\title{
Therapeutic Potential of an Endolysin Derived from Kayvirus S25-3 for Staphylococcal Impetigo
}

\author{
Ichiro Imanishi ${ }^{1,+}$, Jumpei Uchiyama $^{2,+}{ }^{+}$, Toshihiro Tsukui $\left.{ }^{3}{ }^{(}\right)$, Junzo Hisatsune ${ }^{4, \ddagger}$, Kaori Ide ${ }^{1}$, \\ Shigenobu Matsuzaki ${ }^{5}$, Motoyuki Sugai ${ }^{4, \ddagger}$ and Koji Nishifuji ${ }^{1, *(1)}$ \\ 1 Laboratory of Veterinary Internal Medicine, Division of Animal Life Science, Institute of Agriculture, \\ Graduate School, Tokyo University of Agriculture and Technology, 3-5-8 Saiwai-cho, Fuchu, \\ Tokyo 183-8509, Japan \\ 2 Laboratory of Veterinary Microbiology I, School of Veterinary Medicine, Azabu University, \\ 1-17-71 Fuchinobe, Chuo-ku, Sagamihara, Kanagawa 252-5201, Japan \\ 3 Nippon Zenyaku Kogyo Co. Ltd., 1-1 Tairanoue, Sasagawa, Asaka-machi, Koriyama, \\ Fukushima 963-0196, Japan \\ 4 Department of Bacteriology, Graduate school of Biomedical and Health Sciences, Hiroshima University, \\ 1-2-3 Kasumi, Minami-ku, Hiroshima 734-8553, Japan \\ 5 Department of Ophthalmology and Visual Science, Kochi Medical School, Kochi University, 185-1 Kohasu, \\ Oko-cho, Nankoku, Kochi 783-8505, Japan \\ * Correspondence: kojimail@cc.tuat.ac.jp; Tel.: +81-42-367-5777 \\ + These authors contributed equally to this work. \\ $\ddagger$ Present address: Antimicrobial Resistance Research Center, National Institute of Infectious Diseases, \\ 4-2-1 Aoba-cho, Higashimurayama, Tokyo 189-0002, Japan.
}

Received: 22 July 2019; Accepted: 20 August 2019; Published: 22 August 2019

\begin{abstract}
Impetigo is a contagious skin infection predominantly caused by Staphylococcus aureus. Decontamination of $S$. aureus from the skin is becoming more difficult because of the emergence of antibiotic-resistant strains. Bacteriophage endolysins are less likely to invoke resistance and can eliminate the target bacteria without disturbance of the normal microflora. In this study, we investigated the therapeutic potential of a recombinant endolysin derived from kayvirus S25-3 against staphylococcal impetigo in an experimental setting. First, the recombinant S25-3 endolysin required an incubation period of over 15 minutes to exhibit efficient bactericidal effects against $S$. aureus. Second, topical application of the recombinant S25-3 endolysin decreased the number of intraepidermal staphylococci and the size of pustules in an experimental mouse model of impetigo. Third, treatment with the recombinant S25-3 endolysin increased the diversity of the skin microbiota in the same mice. Finally, we revealed the genus-specific bacteriolytic effect of recombinant S25-3 endolysin against staphylococci, particularly S. aureus, among human skin commensal bacteria. Therefore, topical treatment with recombinant S25-3 endolysin can be a promising disease management procedure for staphylococcal impetigo by efficient bacteriolysis of $S$. aureus while improving the cutaneous bacterial microflora.
\end{abstract}

Keywords: Staphylococcus aureus; bacteriophage; endolysin; kayvirus; cutaneous microbiome; impetigo

\section{Introduction}

Impetigo, a bacterial skin infection, is highly contagious among patients and mainly affects children [1]. Over 162 million children have been reported to have suffered from impetigo [2]. Impetigo is generally categorized into two clinical phenotypes, bullous and non-bullous impetigo, and Staphylococcus aureus is a major pathogen in both phenotypes [3]. After the pustules rupture, 
S. aureus can be transmitted to other body sites or other people [3,4]. As a result of the infection process, outbreaks of impetigo are reported in health care facilities and nursery schools [5]. Thus, effective treatment and prevention of bacterial transmission are crucial for the management of impetigo.

In patients with impetigo, topical or systemic antibiotics are recommended as the first-line choice for treatment [3]. Systemic antibiotics are frequently used when lesions become larger, and topical medications become impractical $[3,6]$. However, the frequent use of antibiotics has led to the emergence of new drug-resistant bacteria [6,7], which has recently been considered a major threat to public health $[1,6]$. Antibiotic therapy also disturbs the normal skin microflora [8]. Such medication eliminates other useful commensal bacteria that protect the skin from virulent bacteria $[6,8]$. Thus, new interventions that are less prone to elicit resistance and specifically kill virulent $S$. aureus are needed as an alternative to antibiotics.

Bacteriophage (phage) therapy has received attention as an alternative method to treat drug-resistant bacteria $[9,10]$. Instead of the phage itself, the use of endolysin (a phage-encoded bacteriolytic enzyme that degrades bacterial peptidoglycans) has been attempted to provide a therapeutic agent for various bacterial infections [11,12]. Because of the narrow target spectrum of endolysin, it is considered to eliminate specific bacteria without disturbing the normal bacterial microflora $[10,13,14]$. Several studies have reported that the development of endolysin resistance is less likely than the development of antibiotic resistance $[9,10,15]$. The administration of endolysin has been shown to be effective against bacteremia and pneumonia caused by $S$. aureus in mice $[15,16]$. Because of its effectiveness against $S$. aureus, an endolysin cream has been authorized as a class 1 medical device in Europe [17]. However, the efficacy of endolysin is not always adequate for the treatment of bacterial infections in humans [18]. Thus, the efficacy of phage therapy should be thoroughly and carefully studied before clinical use.

Among the endolysins isolated from Staphylococcus phages, those derived from the family Herelleviridae genus kayvirus are the most well studied $[9,10]$. The endolysin protein sequences are highly conserved among different viral strains belonging to the kayvirus genus [10,19]. The kayvirus endolysins show bacteriolytic and bactericidal activity against Staphylococcus spp. including S. aureus $[10,13,14]$. Although the clinical examination of patients with skin infections, atopic dermatitis, and bacteremia that have been treated with endolysin as an anti-staphylococcal therapy have been conducted [20,21], the efficacy against impetigo, a common skin problem in humans, has not been sufficiently investigated. To apply kayvirus endolysins for the management of impetigo, investigations into the compatibility of endolysin to the management of impetigo in an experimental setting are required.

We previously reported another kayvirus, designated S25-3 [22,23]. In this study, we investigated whether an endolysin derived from kayvirus S25-3 could be used to manage experimental staphylococcal impetigo.

\section{Materials and Methods}

\subsection{Production and Purification of Recombinant Protein}

Phage genomic DNA was obtained as previously described [22]. Based on the available genetic information for kayvirus S25-3 (GenBank accession No.: YP_008854201), primer sets for the S25-3 endolysin gene were designed. After amplification of the genomic DNA by PCR with the primer set (5'-CCCCGGTACCATGGCTAAGACTCAAGCAGAAATAAATAAACG-3' and 5'-CCCCAAGCTTCTAATGATGATGATGATGATGCTTGAATACTCCCCAAGCAACACCA-3'), the DNA fragment with the target sequence was subcloned into the pCold III vector (Takara Bio, Shiga, Japan), resulting in plasmid pCold III S25-3LYS. Plasmid pCold III was used as a negative control. Plasmids pCold III S25-3LYS and pCold III were transformed into Escherichia coli NiCo21 (DE3) (New England BioLabs, Ipswich, MA, USA) and the proteins were overexpressed in accordance with the manufacturer's instructions. E. coli NiCo21 (DE3) clones were cultured in Luria-Bertani broth (LB broth, Miller; Nacalai Tesque, Kyoto, Japan) supplemented with $100 \mu \mathrm{g} / \mathrm{mL}$ ampicillin. 
Bacterial cells were sonicated in a sonication solution (100 mM sodium phosphate, $300 \mathrm{mM}$ $\mathrm{NaCl}, \mathrm{pH}$ 7.8) and the cell lysate was incubated with Co2+-agarose resin (ProteNova, Kagawa, Japan) overnight at $4{ }^{\circ} \mathrm{C}$. After the resin was washed with the sonication solution, the proteins were eluted with elution buffer ( $50 \mathrm{mM}$ sodium phosphate, $300 \mathrm{mM} \mathrm{NaCl}$, pH 7.8 supplemented with $5 \mathrm{mM}$ or $350 \mathrm{mM}$ imidazole). The eluates with $350 \mathrm{mM}$ imidazole contained the recombinant proteins. The eluates with $350 \mathrm{mM}$ imidazole derived from NiCo21 (DE3) [pCold III S25-3LYS], and the corresponding fractions derived from NiCo21 (DE3) [pCold III], were dialyzed against phosphate-buffered saline (PBS; pH 7.4). After separation of the proteins by SDS-PAGE and staining with Coomassie G-250 solution (Life Technologies, Carlsbad, CA, USA), the protein integrity was confirmed. The protein concentration of S25-3LYS-his was measured by a BCA kit (Thermo Fisher Scientific, Waltham, MA, USA). After dialysis, the fraction obtained from NiCo21 (DE3) [pCold III], corresponding to the fraction containing S25-3LYS-his, was used as a negative control solution.

\subsection{Bacterial Strains}

S. aureus strains COL [24], N315 [25], TY767, TF3030 [26], TY825 [27], N1 [28], and N2 [28] were used to examine the bacteriolytic and bactericidal activities of S25-3LYS-his. S. aureus strain TY825, which is a methicillin-resistant $S$. aureus strain producing exfoliative toxin $B$, a virulent factor of bullous impetigo [27], was used for topical inoculation of the mouse pinnae.

Staphylococcus epidermidis strains ATCC12228 [24], W860371, and M890190, Streptococcus mitis strain GTC495 [29], and Pseudomonas aeruginosa strains MS5639, MS5640, MS5641, PAO1 [30], D4 [30], S10 [30], and PA29 [30] were used to measure the lytic spectrum of the endolysin. The human commensal skin bacteria were also isolated as described below (see Table S2 in the supplemental material).

\subsection{Bacterial Isolation from Human Skin}

Twelve human volunteers, including four females and eight males, between 24 and 49 years old, were included in this study. Skin swabs were taken from three anatomic sites, including the forehead, the flexor part of the left forearm, and the dorsal skin. At each site, a $1 \times 1-\mathrm{cm}$ square of the skin surface was swabbed 30 times using a sterile cotton swab with constant pressure. These volunteers were informed of the study's goals and provided written consent for participation before they were included in the study. All experiments using human resources were approved by the Ethical Committee at the Tokyo University of Agriculture and Technology (\#30-04).

The bacteria were isolated from the swabs by growth on three culture media: tryptic soy agar (Beckton Dickinson and Co. Franklin Lakes, NJ, USA), Brucella HK medium (Kyokuto Pharmaceutical Industrial, Tokyo, Japan), and Hoyle's tellurite agar (Beckton Dickinson and Co.). Bacteria were cultured on tryptic soy agar at $35^{\circ} \mathrm{C}$ for 3 days under aerobic conditions, on Brucella HK medium at $37^{\circ} \mathrm{C}$ for 3 days under anaerobic conditions, and on Hoyle's tellurite agar at $37^{\circ} \mathrm{C}$ for 5 days under aerobic conditions. Purification was performed at least three times. After the purification procedure, the bacterial cells were collected from the agar plates for phylogenetic classification and bacteriolytic assessment.

\subsection{Phylogenetic Classification of Bacteria}

After isolating the bacterial DNA, bacterial 16S rDNA fragments were amplified by PCR using a Bacterial 16S rDNA PCR Kit (Takara Bio). PCR products were sequenced with the BigDye Terminator v3.1 cycle sequencing kit (Life Technologies) using a Model 3130 Genetic Analyzer (Life Technologies), according to the manufacturer's instructions. Sequences were phylogenetically assessed using the Ribosomal Database Project (RDP) (https://rdp.cme.msu.edu/) [31] and BLASTp at the National Center for Biotechnology Information (https://blast.ncbi.nlm.nih.gov/Blast.cgi). 


\subsection{Measurement of the Bacteriolytic and Bactericidal Activities}

The bacteriolytic activity of S25-3LYS-his was assessed. Bacteria were cultured on appropriate media (see Table S2 in the supplemental material). Bacterial cells were washed and resuspended in sterile PBS containing $5 \mathrm{mM} \mathrm{NaCl}$. The optical density was adjusted to $\sim 1.0$ at $600 \mathrm{~nm}$ using a spectrophotometer (Bio Spectrometer; Eppendorf, Hamburg, Germany). Then, $195 \mu \mathrm{L}$ of S. aureus suspension was supplemented with $5 \mu \mathrm{L}$ of S25-3LYS-his $(400 \mu \mathrm{g} / \mathrm{mL})$ or control solution as a negative control in a flat-bottomed 96-well microplate (Life Technologies). Preliminary studies revealed that the above concentrations of S25-3LYS-his and S. aureus suspension yielded the most effective bacteriolytic activity against $S$. aureus. The microplate was incubated for an appropriate period at $37^{\circ} \mathrm{C}$, and the optical density at $595 \mathrm{~nm}$ was measured over time using an iMark plate reader (BioRad Laboratories, Hercules, CA, USA). The reaction rate $\left(\Delta \mathrm{OD}_{595} / \mathrm{min} / \mathrm{mg}\right)$ was calculated using a formula described in Figure $\mathrm{S} 2$ in the supplemental material.

The bactericidal activity of S25-3LYS-his was also assessed. Bacteria were cultured to mid-log phase, then $10 \mu \mathrm{L}$ of endolysin $(1 \mathrm{mg} / \mathrm{mL})$ or control solution was added to $190 \mu \mathrm{L}$ of bacterial suspension $\left(1.6 \times 10^{8} \mathrm{CFU} / \mathrm{mL}\right)$. After incubation at $37^{\circ} \mathrm{C}$ for the appropriate period, the bacterial suspension was plated on mannitol salt agar (Nissui Pharmaceuticals, Tokyo, Japan). After incubation $\left(37^{\circ} \mathrm{C}\right.$ for $\left.48 \mathrm{~h}\right)$, the viable bacteria were counted.

The rate of turbidity reduction $\left(\Delta \mathrm{OD}_{595}\right)$, bacterial reduction $(\triangle \mathrm{CFU} / \mathrm{mL})$, and the bacteriolytic reaction rate within $15 \mathrm{~min}\left(\Delta \mathrm{OD}_{595} / \mathrm{min} / \mathrm{mg}\right)$ were calculated using a formula described in Figure $\mathrm{S} 2$ in the supplemental material.

\subsection{Application of S25-3LYS in a Mouse Model of Impetigo}

S. aureus strain TY825 was cultured in LB broth (LB Broth, Miller; Nacalai Tesque) until mid-log-phase, and was then washed with PBS three times. Six-week-old, female, BALB/c Cr Slc mice (Sankyo Labo Service, Tokyo, Japan) were used. After tape-stripping was performed on the murine inner pinnae seven times, a $1 \times 1-\mathrm{cm}$ gauze containing $2.0 \times 10^{8} \mathrm{CFU}$ of $S$. aureus was adhered to the right and left pinnae. Two microliters of S25-3LYS-his $(1 \mathrm{mg} / \mathrm{mL})$ and the same volume of control solution were applied to the gauze attached to the right and left pinnae, respectively. Six hours after inoculation, the mice were euthanized by cervical dislocation, and the pinnae were collected for further experiments.

The animal experiment was approved by the Animal Research Committee at Tokyo University of Agriculture and Technology (\#27-40) and was performed under the International Guiding Principles for Biomedical Research Involving Animals.

\subsection{Enumeration of Bacterial Densities in the Mouse Pinnae}

Mouse pinnae were suspended and mixed in $500 \mu \mathrm{L}$ of PBS, and the suspension was plated onto mannitol salt agar. After incubation $\left(37^{\circ} \mathrm{C}\right.$ for $48 \mathrm{~h}$ ), the appearance of bacterial colonies was enumerated.

\subsection{Histopathological and Immunofluorescence Analyses}

The mouse pinnae tissues were fixed with $10 \%$ formaldehyde $(N=6$, each group). Each tissue was dissected into 10 pieces and embedded in paraffin. After paraffin-embedded samples were serially sectioned, the sections were deparaffinized. For histopathological analysis, tissue sections were stained with hematoxylin and eosin and then observed.

For immunofluorescence analysis, the deparaffinized sections were incubated with primary and then secondary antibodies. As primary antibodies, mouse anti-human keratin type 1 and 2 monoclonal antibody (clone AE1+AE3; Progen Biotechnik GmbH; Heidelberg, Germany) and rabbit anti-Staphylococcus polyclonal antibody (clone CH91; courtesy of Makoto Haritani, National Institute of Animal Health, Ibaraki, Japan) [32] were used. As secondary antibodies, Alexa Fluor ${ }^{\mathrm{TM}}$ 546-conjugated 
goat anti-rabbit IgG and Alexa Fluor ${ }^{\mathrm{TM}} 488$ goat anti-mouse IgG (Life Technologies) were used. Nuclei were counterstained with Hoechst 33258 (Life Technologies).

Tissue sections were observed using a light and fluorescence microscope (BX43 F; Olympus, Tokyo, Japan). Micrographic images were recorded using image software (CellSens Standard; Olympus). The length of the basement membrane (BM), the dimensions and number of intra-epidermal clefts, and the number of intra-epidermal staphylococci, were quantified by previously described methods [28].

\subsection{Microbiota Analysis}

The pinnae tissues were suspended in $500 \mu \mathrm{L}$ of sterile PBS and mixed. After dilution, $100 \mu \mathrm{L}$ of the pinna solution was plated onto tryptic soy agar and incubated at $37^{\circ} \mathrm{C}$ for $48 \mathrm{~h}$ under aerobic conditions. Ten colonies per pinna were randomly isolated. Phylogenetic classification of the bacteria was performed by sequencing the $16 \mathrm{~S}$ rRNA gene as described above.

\subsection{Microbiome Analysis}

The DNA was purified directly from tissue samples obtained from the mouse pinnae using an ISOSPIN Fecal DNA kit (Nippon Gene, Toyama, Japan), in accordance with the manufacturer's instructions. The V3-V4 region of the 16S rRNA gene [33] was amplified from $500 \mathrm{ng}$ of extracted DNA using the optimized methods for Illumina MiSeq (Illumina, San Diego, CA, USA) and barcoded sample-specific primers (forward, 5'-TCGTCGGCAGCGTCAGATGTGTATAAGAG ACAGCCTACGGGNGGCWGCAG-3'; reverse, 5'-GTCTCGTGGGCTCGGAGATGTGTATAAGAGAC AGGACTACHVGGGTATCTAATCC-3'). Each PCR product was purified using an Agencourt AMPure XP Beads Kit (Beckman Coulter, Pasadena, CA, USA) and quantified using a Qubit dsDNA BR Assay Kit (Thermo Fisher Scientific). Each 100-ng amplicon underwent a second round of PCR for indexing, using a Nextera XT Index Kit v2 (Illumina). After purification, the PCR products were quantified by NanoPhotometer (Implen, Westlake Village, CA, USA) and pooled into one tube with a final concentration of $1.6 \mathrm{ng} / \mu \mathrm{L}$. The concentration of the pooled DNA library was validated using an Agilent 2100 Bioanalyzer (Agilent, Santa Clara, CA, USA). After denaturation with $\mathrm{NaOH}, 850 \mu \mathrm{L}$ of a 9 pM DNA library and $150 \mu \mathrm{L}$ of $9 \mathrm{pM}$ PhiX were mixed and subjected to pair-end sequencing using Illumina MiSeq with a MiSeq Reagent Kit v3 (Illumina).

The sequence data were analyzed using Quantitative Insights into Microbial Ecology 2 (QIIME2) v2019.1.0 [34]. The DADA2 software package v2018.4.0 incorporated in QIIME 2 was used to correct amplicon sequence errors and to construct an amplicon sequence variant table [35]. The Greengenes $99 \%$ reference database v13.8 was used for the taxonomic classification of each amplicon sequence variant. The microbiome diversity was analyzed using a rarefied amplicon sequence variant table.

\subsection{Statistical Analysis}

Statistical analysis was performed using GraphPad Prism 6 (GraphPad Software Inc., San Diego, CA, USA). Comparisons of bacterial turbidity and bacterial reduction between control and S25-3LYS-his groups were performed using the Mann-Whitney U test. Correlations between turbidity reduction and bacterial reduction in S25-3LYS-his-treated groups were analyzed by Spearman's correlation coefficient, and the least square regression line was calculated. The density of staphylococcal cells, number of staphylococcal cells in intra-epidermal tissue, and area and frequency of pustules in mouse skins were analyzed using the Mann-Whitney $U$ test. The taxonomic abundance of viable bacteria from murine pinnae and the frequency of bacteria showing "sensitivity" in commensal bacteria on human skins were analyzed using the Chi-square test. Reaction rate of "sensitive" staphylococci by S25-3LYS-his were analyzed using the Kruskal-Wallis test.

Metrics of alpha diversity (i.e., Faith-Phylogenetic Diversity (PD), Chao1, and Shannon) and beta diversity (i.e., weighted and unweighted UniFrac) were examined using QIIME 2 [34]. The correlations of the metrics of alpha and beta diversities with age were statistically analyzed by Kruskal-Wallis and PERMANOVA tests, respectively, implemented in QIIME 2 [34]. 


\section{Results and Discussion}

\subsection{Characteristics of the Endolysin Derived from Kayvirus S25-3}

Amino acid sequence analysis revealed that the endolysin derived from kayvirus S25-3 shared 99.39\% identity with the endolysin derived from kayvirus K, the first-isolated kayvirus endolysin [36]. The endolysin gene of kayvirus S25-3 was subcloned into a protein expression vector and transferred into $E$. coli to generate a recombinant endolysin fused with a $6 \times$ histidine tag at the C-terminus. This recombinant endolysin is termed S25-3LYS-his in this study. First, mass spectrometry analysis revealed that the cleavage sites of the $S$. aureus peptidoglycan by S25-3LYS-his were identical to those digested by endolysins of other kayviruses K, GH15, and SAP-1 (see Figure S1 in the supplemental material) $[10,37,38]$. Second, in vitro incubation of S25-3LYS-his with nine clinical isolates of S. aureus revealed that the reduction of bacterial turbidity and the bactericidal effects of S25-3LYS-his were observed within 15 min of incubation (see Table S1 in the supplemental material), which was similar to previous reports of kayvirus endolysins [11,37,38]. The purified S25-3LYS-his was used for the following studies. The purified solution prepared from E. coli harboring the protein expression vector without inserted DNA was used as a negative control.

\subsection{Prolonged Incubation of S25-3 Endolysin with S. aureus Increases the Bactericidal Effect}

Because endolysins have been considered to be rapid-acting antimicrobial agents $[11,15,37,38]$, most of the previous studies examined the bacteriolytic and bactericidal effects of endolysins over a short time period. However, to apply endolysins as topical pharmaceutical agents for controlling bacterial skin infections, it is important to understand the appropriate exposure period of endolysins to obtain maximum bactericidal effects. Thus, we compared the bacteriolytic and bactericidal effects of S25-3LYS-his against $S$. aureus following application over short and long time periods.

First, we measured the changes in bacterial turbidity and the number of viable bacteria after $15 \mathrm{~min}$ and $360 \mathrm{~min}$ of incubation with S25-3LYS-his (Figure 1A). The reduction rate of bacterial turbidity and the number of viable bacteria $\left(\triangle \mathrm{OD}_{595}\right.$ and $\left.\triangle \mathrm{CFU} / \mathrm{mL}\right)$ following S25-3LYS-his treatment were calculated using the formula described in Figure S2A and S2B in the supplemental materials, respectively. The correlations between the reduction rates of bacterial turbidity and the numbers of viable bacteria at $15 \mathrm{~min}$ and $360 \mathrm{~min}$ were also analyzed.

Our results revealed that the rate of turbidity reduction was not statistically correlated with the reduction of viable bacteria after 15 min of incubation $(P>0.05$; Figure $1 \mathrm{~B})$. However, this correlation was observed after $360 \mathrm{~min}$ of incubation $(P<0.05$; Figure $1 \mathrm{~B})$. This result suggested that a shorter exposure to S25-3LYS-his exhibited insufficient bactericidal activity against $S$. aureus strains, but efficient bacteriolytic activity. It is assumed that some populations of $S$. aureus become protoplasts following short exposure to S25-3LYS-his, as observed with other peptidoglycan-degrading enzymes such as lysozyme [39].

As bacteriolytic-bactericidal correlations can be represented as a regression line, we compared the bacteriolytic-bactericidal regression lines obtained after $15 \mathrm{~min}$ and $360 \mathrm{~min}$ of incubation. A higher slope of the regression line was associated with stronger bactericidal effects during constant bacteriolytic activity. The slope of the regression line at $360 \mathrm{~min}$ of incubation was 20.31, while that at 15 min of incubation was 4.43 (Figure 1B). These findings indicated that prolonged incubation with S25-3LYS-his increased the bactericidal effect against $S$. aureus. 

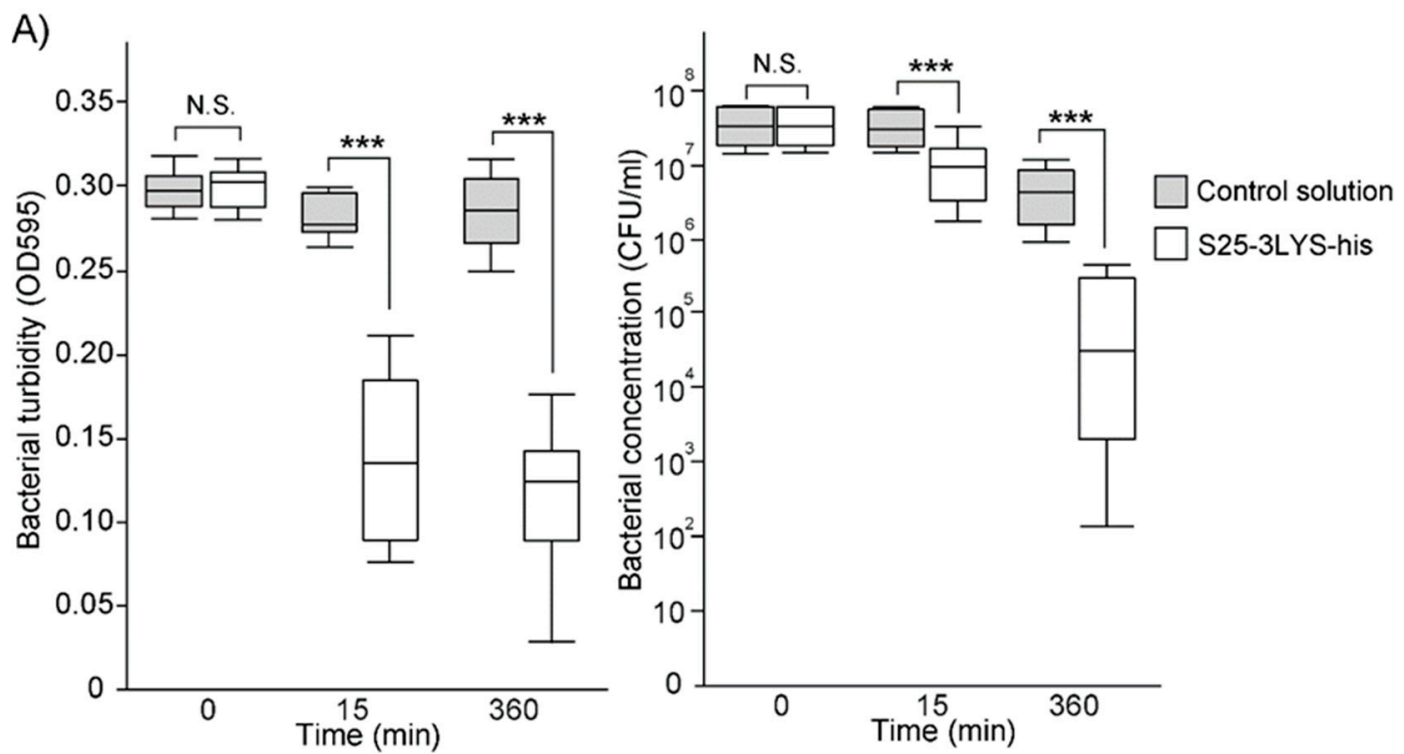

B)

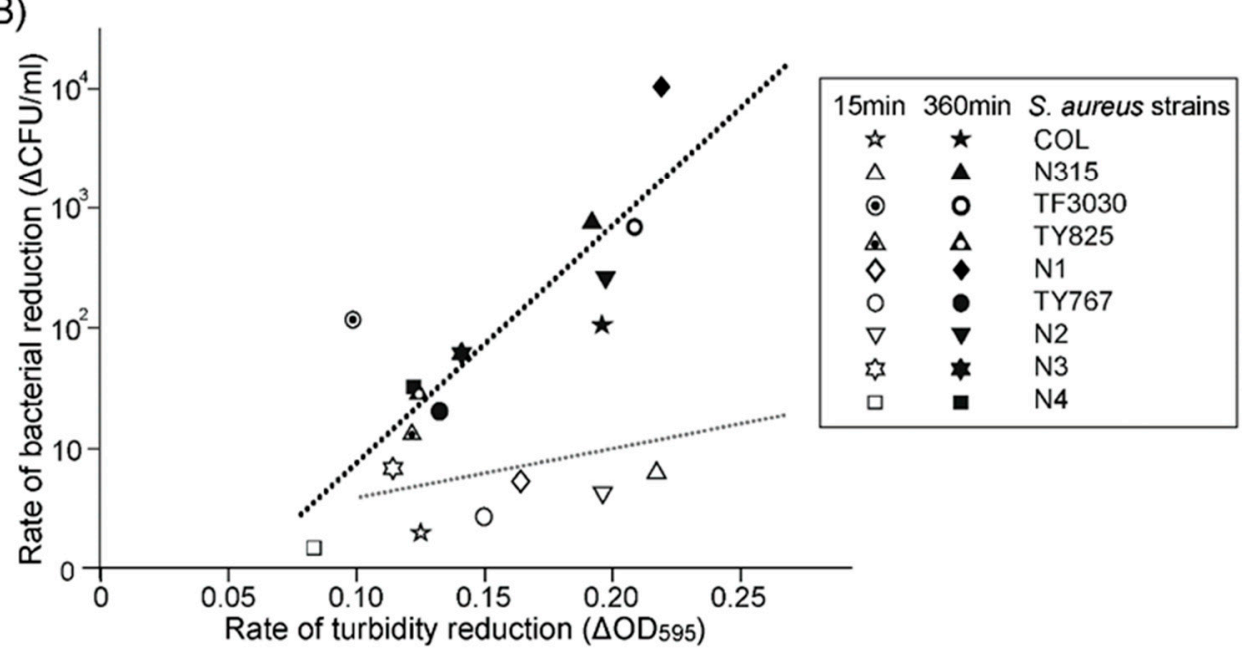

Figure 1. Description of S25-3LYS-his activities against S. aureus. (A) Comparison of bacterial turbidity (left) and bacterial reduction (right) between the control and S25-3LYS-his groups. At 15 min of incubation, bacterial turbidities $\left(\mathrm{OD}_{595}\right)$ of the control and S25-3LYS-his groups were $0.284 \pm 0.013$ and $0.141 \pm 0.005$, respectively (mean $\pm \mathrm{SD}$ ). At $360 \mathrm{~min}$ of incubation, the bacterial turbidities of the control and S25-3LYS-his groups were $0.286 \pm 0.023$ and $0.115 \pm 0.045$, respectively. At $15 \mathrm{~min}$ of incubation, the bacterial concentrations $\left(\log _{10} \mathrm{CFU} / \mathrm{mL}\right)$ of the control and S25-3LYS-his groups were $7.41 \pm 0.24$ and $6.80 \pm 0.39$, respectively. At $360 \mathrm{~min}$ of incubation, the bacterial concentrations of the control and S25-3LYS-his groups were $7.41 \pm 0.24$ and $6.80 \pm 0.39$, respectively. Statistical significance is indicated as "**** $(P<0.005)$; no statistical significance is indicated as "N.S.". (B) Correlation analysis between turbidity reduction and bacterial reduction in the S25-3LYS-his-treated groups, measured at 15 and $360 \mathrm{~min}$. The regression lines are separately plotted around the data points at $15 \mathrm{~min}\left(R^{2}=0.22\right)$ and $360 \mathrm{~min}\left(R^{2}=0.76\right)$, which are shown in gray and black, respectively. The $S$. aureus strains tested are shown in the right-hand box.

\subsection{Topical Application of S25-3 Endolysin Inhibits Staphylococcal Epidermal Invasion in Experimental Staphylococcal Impetigo}

We previously generated a mouse model of staphylococcal impetigo by topical inoculation of S. aureus strains onto tape-stripped mouse skin [28]. In the mouse model, staphylococci adhere to the viable epidermis and recruit neutrophils, then penetrate into the epidermis through inter-keratinocyte clefts created by the neutrophils. In this study, we investigated the therapeutic effect of S25-3LYS-his in 
this experimental mouse model of impetigo. The S25-3LYS-his was applied onto tape-stripped murine pinnae after inoculation of the same area with S. aureus. At $6 \mathrm{~h}$ after inoculation, murine pinnae were collected, and the densities of staphylococci between the S25-3LYS-his-treated group and the control group were compared. The S25-3LYS-his-treated group showed a lower concentration of staphylococci than the control group (mean \pm standard deviation, $4.69 \pm 0.69 \log _{10} \mathrm{CFU} /$ tissue in S25-3LYS-his-treated group and $5.44 \pm 0.46 \log _{10} \mathrm{CFU} /$ tissue in control group, $P<0.01$; Figure $2 \mathrm{~A}$ ).

The tissues were also subjected to immunofluorescence analysis. Staphylococci were observed in intraepidermal clefts in the control group but were only detected on the skin surface in the S25-3LYS-his-treated group. The numbers of intraepidermal staphylococci per $1 \mathrm{~mm}$ of the BM in the S25-3LYS-his-treated group were lower than those in the control group $(0.24 \pm 0.06$ bacteria $/ \mathrm{mm}$ of BM in S25-3LYS-his-treated group and $2.07 \pm 0.90$ bacteria/mm of BM in control group, $P<0.005$; Figure 2C). These results indicated that topical application of S25-3LYS-his inhibited S. aureus invasion of the epidermis in the experimental mouse model of impetigo.

The area of intraepidermal pustules and the frequency of pustules per $1 \mathrm{~cm}$ of BM were also analyzed in skin sections. The area of pustules in the S25-3LYS-his-treated group was smaller than in the control group $\left(232.6 \pm 40.9 \mu \mathrm{m}^{2}\right.$ in S25-3LYS-his-treated group and $391.7 \pm 132.0 \mu \mathrm{m}^{2}$ in control group, $P<0.05$; Figure $2 \mathrm{C}$ ), whereas the frequency of pustules was not statistically different between the two groups $(1.90 \pm 1.26 / \mathrm{cm}$ BM in S25-3LYS-his-treated group and $2.08 \pm 0.64 / \mathrm{cm} \mathrm{BM}$ in control group, $P>0.05$; Figure 2C). These results indicated that S25-3LYS-his suppressed the enlargement of pustules in the mouse model of impetigo, although it did not inhibit pustule formation. 


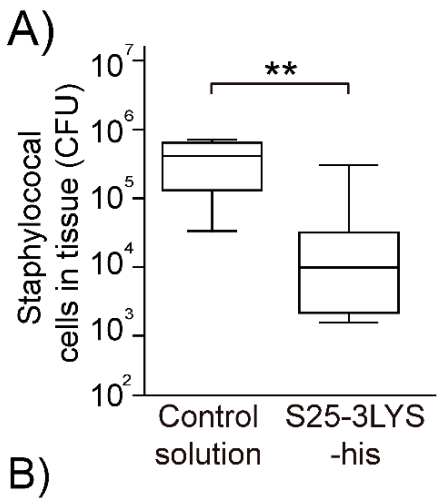

B)

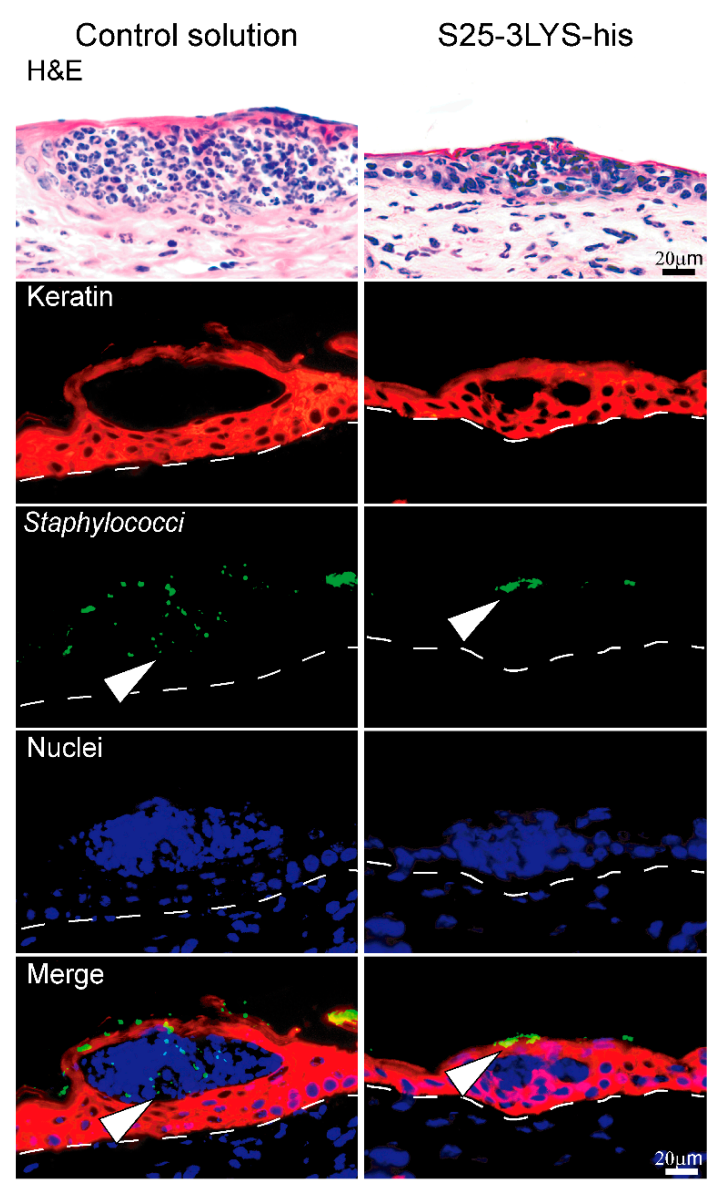

C)

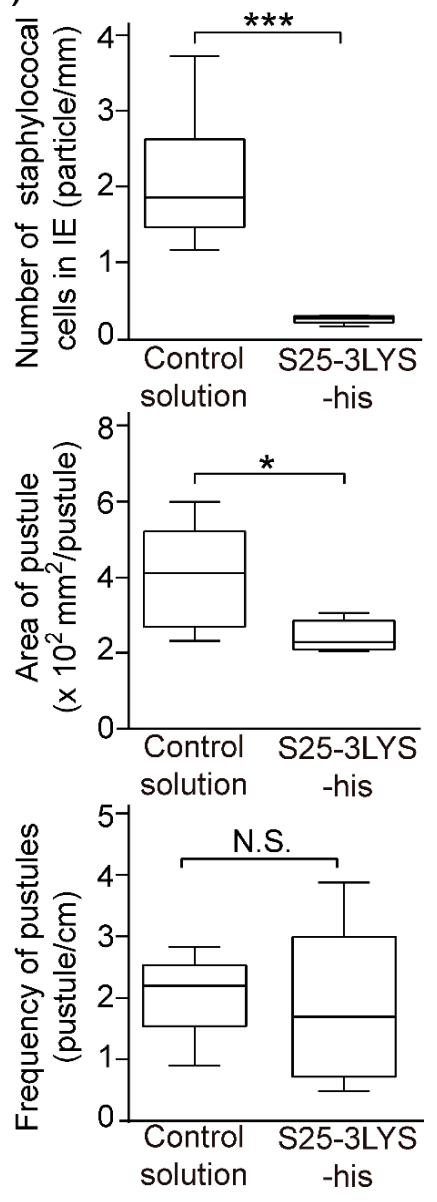

Figure 2. Evaluation of S25-3LYS-his treatment in an impetigo mouse model. (A) The density of staphylococcal cells in mouse skin. The densities of viable Staphylococcus spp. were measured using mannitol salt agar. The statistical significance is indicated as "**" $(P<0.01)$. Of note, when the total bacteria in the mouse skin was measured using tryptic soy agar, there was no statistical difference between the S25-3LYS-his-treated and control groups $(P>0.05$; see Figure S4 in the supplemental material). (B) Pathological analysis of mouse skin tissues in the control solution (left) and S25-3LYS-his-treated solution (right). Representative photographs are shown. In the top micrographs, the tissues stained with hematoxylin and eosin (H\&E) are shown. In the second from the top to the bottom micrographs, keratin, staphylococci, and nuclei-specific staining are shown in red, green, and blue, respectively. Staphylococci are indicated by white arrowheads (i.e., images on the third and the fifth rows from the top). The dotted lines indicate the basement membranes. (C) Staphylococcal cells in the intra-epidermal (IE) region, the area of pustules, and the frequency of pustules were measured by pathological analysis. Statistical significance is shown on the graph $\left({ }^{*}, P<0.05 ;{ }^{* * *}, P<0.005\right)$; no statistical significance is indicated as "N.S." 


\subsection{S25-3LYS-his Treatment Increases Bacterial Diversity in Mouse Skin}

Because kayvirus endolysins are thought to lyse Staphylococcus spp. selectively [40], restoration of the microbial balance on skin infected by staphylococci may be achievable by the application of kayvirus endolysins. Therefore, the skin microbial diversities of the S25-3LYS-his-treated group and the control group were analyzed. We revealed that the taxonomic abundance of viable bacteria, which were harvested from the murine pinnae and cultured on tryptic soy agar, was significantly higher in the S25-3LYS-his treatment group than in the control group $(P<0.05$; Figure $3 \mathrm{~A})$.
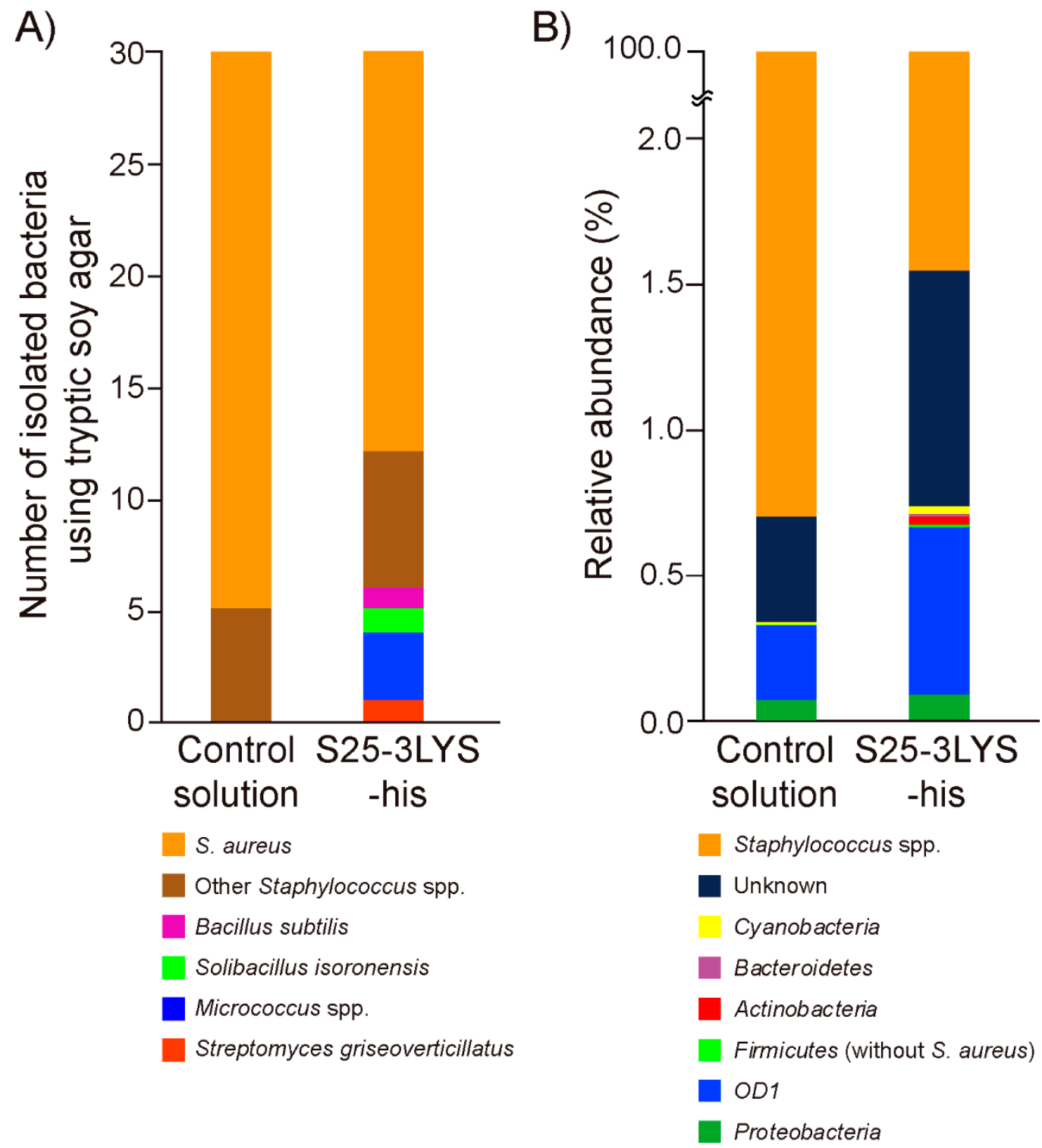

Figure 3. Taxonomical abundance of the microbiome on mouse skin treated with the control solution or S25-3LYS-his, as determined by (A) culture methods and (B) 16S rRNA gene amplicon analysis. At the bottom, bacterial taxonomical assignments are shown. Using the culture method, 30 bacteria were isolated from the skin $(N=3)$ for each treatment group, then phylogenetically classified based on their 16S rRNA gene sequence. Using 16S rRNA gene amplicon analysis, the average taxonomical abundance of the skin microbiome $(N=3)$ for each treatment group is shown. The phylum taxonomical level is shown. For data analysis, a total of 182,317 reads were used, with each sample comprising $30,386.2 \pm 5886.1$ reads (mean $\pm S D$ ), of which 27,700 reads in each sample were rarefied. 
Taxonomic annotation using the bacterial 16S rRNA sequence database revealed that over $98 \%$ of bacteria in both the S25-3LYS-his-treated group and the control group were Staphylococcus spp. (Figure 3B). However, the analysis of alpha diversity revealed that Chao1 and phylogenic diversity (Faith-PD) indexes in the S25-3LYS-his-treated group were significantly higher than the control group $(P<0.05$; see Figure S3 in the supplemental material), whereas there were no statistical differences in the Shannon index between the two groups $(P=0.5127)$. Considering that Chao1 and Faith-PD explain species richness, while Shannon index explains both richness and evenness of the species present, our findings indicate that S25-3LYS-his increased the alpha diversity of the bacterial microbiome on the skin surface, although S. aureus was still predominant in the S25-3LYS-his-treated group.

By contrast, the analysis of beta diversity revealed that there were no statistical differences in the weighted UniFrac and unweighted UniFrac values between the two groups ( $P=0.7040$ and 0.1840 in weighted UniFrac and unweighted UniFrac, respectively). At a minimum, these findings indicated that topical application of S25-3LYS-his increased bacterial richness. It is worth noting that 16S rRNA amplicon analysis could not discriminate between the sequences of dead bacteria and those of viable bacteria, or between $S$. aureus and other staphylococcal species.

\subsection{S25-3 Endolysin Preferentially Lyses S. aureus among Human Skin Bacteria}

We further examined the lytic activity of S25-3LYS-his against commensal bacteria on human skin in vitro. A collection of human skin commensal bacteria was prepared, which included 11 reference strains and 89 originally isolated bacteria from skin swabs of healthy individuals. It has been reported that three genera (Staphylococcus, Corynebacterium, and Propionibacterium) constitute over $60 \%$ of the bacterial species present in the human skin microbiota [41,42]. In addition to these three genera, other bacteria including S. mitis, P. aeruginosa, Bacillus spp., Micrococcus spp., and Brevibacterium spp. were also isolated in our study (see Figure $\mathrm{S} 2$ in the supplemental material).

Bacterial sensitivity was determined by comparing bacterial turbidity between the S25-3LYS-his-treated group and the control group after $360 \mathrm{~min}$ of incubation. In this experiment, "sensitivity" meant that bacterial turbidity in the S25-3LYS-his-treated group was significantly lower than in the control group. By contrast, "insensitivity" meant that there were no statistical differences in turbidity between the two groups. We found that the frequency of bacteria showing "sensitivity" was significantly higher in S. aureus strains than in S. epidermidis strains $(P<0.005)$ and other staphylococcal strains $(P<0.01$; Figure $4 \mathrm{~A})$.

Moreover, the bacteriolytic reaction rate of "sensitive" staphylococci induced by S25-3LYS-his $\left(\triangle \mathrm{OD}_{595} / \mathrm{min} / \mathrm{mg}\right.$ S25-3LYS-his; see Figure S2C in the supplementary material) was examined. The reaction rates were significantly higher in S. aureus strains $(N=21)$ than in Staphylococcus epidermidis strains $(N=5, P<0.005)$ and other staphylococcal strains $(N=7, P<0.005$; Figure $4 \mathrm{~B})$. These findings indicated that S25-3LYS-his preferentially lysed S. aureus among human skin commensal bacteria. 

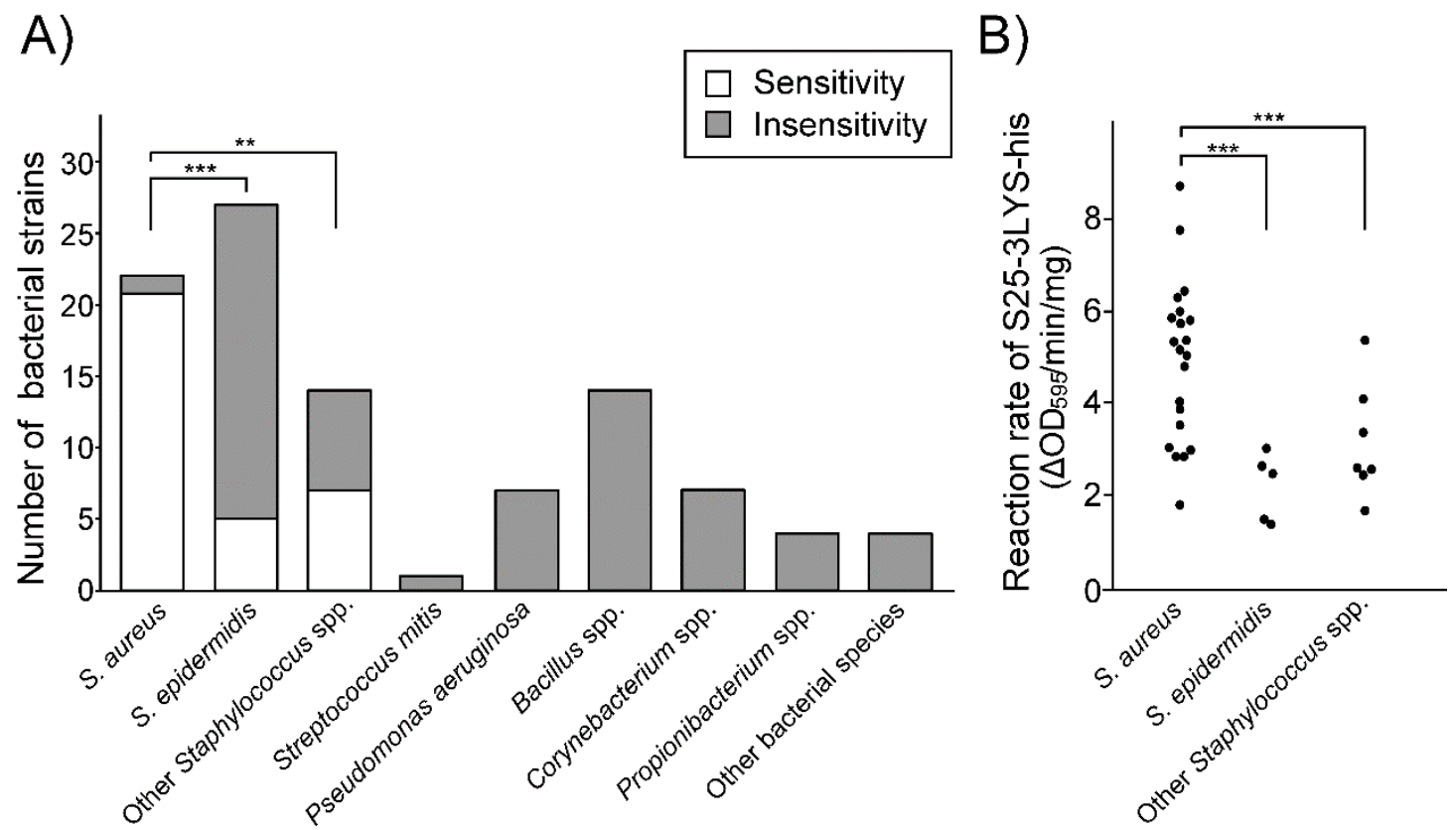

Figure 4. Lytic spectra of S25-3LYS-his on cutaneous bacteria. (A) Only staphylococci showed sensitivity to S25-3LYS-his. Strains showing "sensitivity" and "insensitivity" to S25-3LYS-his are shown in white and black squares, respectively. (B) Bacteriolytic reaction rates of S25-3LYS-his $\left(\triangle \mathrm{OD}_{595} / \mathrm{min} / \mathrm{mg}\right)$ to Staphylococcus spp. showing sensitivity to S25-3LYS-his. Statistical significance is shown on the graph $\left({ }^{*}, P<0.05 ; * *, P<0.01 ; * *, P<0.005\right)$.

\subsection{Future Prospects for Endolysin Application to Treat Staphylococcal Skin Infections}

In the present study, we showed that topical application of a recombinant endolysin derived from kayvirus S25-3 suppressed the enlargement of pustules and percutaneous invasion of staphylococci in an experimental model of impetigo. Further, the recombinant S25-3 endolysin preferentially decontaminated $S$. aureus with minimal effects on other skin commensal bacteria. Considering the similarities between S25-3 endolysin and other kayvirus endolysins in protein sequence and antimicrobial properties, our findings suggest that kayvirus endolysins have therapeutic potential for impetigo. To introduce kayvirus endolysin as a pharmaceutical agent for impetigo management successfully, further examination of the productization and safety of the endolysins should be considered.

For productization, the endolysin requires optimization as a pharmaceutical agent with good usability. First, because the topical application of endolysin over an extended period is expected to exhibit maximal bactericidal effects, an appropriate carrier material is needed for maintenance of endolysin on the skin. Second, considering the dissemination of the endolysin product, the endolysin needs to show decontamination effects superior to those of conventional agents. kayvirus endolysins are too large to penetrate the viable epidermis $[43,44]$ and water-soluble proteins have difficulty penetrating the lipid bilayer in the stratum corneum; both of these factors present challenges in accessing S. aureus in the tissues. To effectively control the infection, the permeability of endolysin needs to be improved, potentially by developing a strategy of efficient transdermal drug delivery. Third, although endolysins are highly stable in general $[45,46]$, the stability ofkayvirus endolysin combined with carrier materials therefore needs to be examined [47] to ensure that other components of the endolysin product do not disable its stability. Fourth, as some cases of non-bullous impetigo are caused by Streptococcus pyogenes and endolysins specific to Streptococcus spp. have been reported [48], the latter endolysins should be combined with kayvirus endolysins for the effective treatment of impetigo.

The safety of the long-term usage of topical endolysin products needs to be investigated. First, kayvirus endolysin is generally recognized as a safe agent because no adverse effects have been reported 
in pre-clinical and clinical trials $[37,49,50]$. In addition, a recent clinical trial revealed that systemic administration of an endolysin was effective for controlling $S$. aureus bacteremia in humans [49]. However, because the safety of the long-term usage of topical endolysin has not been confirmed, we believe that safety analysis of kayvirus endolysin is required. The repeated exposure to protein on the skin over a long period is thought to increase the risk of hypersensitivity [51]. The production of anti-endolysin antibodies has been shown as a result of topical application of chimeric endolysin in a murine model [52]. Some other lytic enzymes, such as egg lysozyme, have been shown to cause allergic reactions in both humans and dogs $[53,54]$. Endolysin treatment is not expected to lead to the appearance of resistant strains $[13,15]$. However, the long-term use of endolysin in clinical settings may lead to the emergence of endolysin-resistant bacteria.

Our findings suggest that the topical application of kayvirus endolysins to the site of infection is effective for controlling impetigo by decontamination of $S$. aureus while maintaining the robustness of the skin microbiome. In addition to applying kayvirus endolysin for the management of impetigo, endolysin can be applied to decontaminate virulent $S$. aureus from potential carriers. The endolysin cream can be prophylactically applied to individuals at risk of infection in certain settings, e.g., kindergarten or elementary school, where the hands of individuals are most likely to be contaminated with virulent $S$. aureus $[55,56]$. When kayvirus endolysin is topically applied to the hands after washing, it potentially prevents the spread of virulent $S$. aureus to the environment as a result of its efficient bacteriolytic activity and could therefore be used to prevent the spread of virulent $S$. aureus among children. With further study, endolysin can potentially be applied for the management of various staphylococcal skin infections in clinical settings.

Supplementary Materials: The following are available online at http://www.mdpi.com/1999-4915/11/9/769/s1, Table S1: S. aureus strains used in this study, Table S2: Collection of human cutaneous bacteria used in this study, Figure S1: Analysis of S. aureus peptidoglycan reacted with S25-3LYS-his, using liquid chromatography coupled with quadruple time-of-flight mass spectrometry (QTOF), Figure S2: Mathematical formulas for (A) rate of turbidity reduction, (B) rate of bactericidal reduction, and (C) bacteriolytic reaction rate of S25-3LYS-his, Figure S3: Alpha diversity of mouse pinna microbiome, and Figure S4: Bacterial density of mouse pinnae, measured by culture method.

Author Contributions: Conceptualization, J.U. and K.N.; Funding acquisition, I.I., J.U., T.T. and K.N.; Project administration, K.N.; Resources, J.U., J.H. and M.S.; Investigation, I.I. and J.U.; Validation, I.I., J.U.; Supervision, K.I. and S.M.; Writing—original draft, I.I. and J.U.; Writing—review and editing, T.T., J.H., K.I., S.M., M.S. and K.N.

Funding: This research was funded by JSPS KAKENHI, grant numbers JP17H03922 and JP15K19095, and a Grant-in-Aid for a JSPS Research Fellow, grand number JP17J01839.

Acknowledgments: The authors are grateful to Makoto Haritani for providing rabbit anti-Staphylococcus polyclonal antibody. The authors are also grateful to Agilent Inc. for performing LC-Q-TOF MS analysis. We also thank Kate Fox, DPhil, and Ryan Chastain-Gross, PhD, from Edanz Group (www.edanzediting.com/ac) for editing a draft of this manuscript.

Conflicts of Interest: T.T. is an employee at Nihon Zenyaku Kogyo Co. Ltd.

\section{References}

1. D'Cunha, N.M.; Peterson, G.M.; Baby, K.E.; Thomas, J. Impetigo: A need for new therapies in a world of increasing antimicrobial resistance. J. Clin. Pharm. Ther. 2018, 43, 150-153. [CrossRef] [PubMed]

2. Bowen, A.C.; Mahe, A.; Hay, R.J.; Andrews, R.M.; Steer, A.C.; Tong, S.Y.; Carapetis, J.R. The global epidemiology of impetigo: A systematic review of the population prevalence of impetigo and pyoderma. PLoS ONE 2015, 10, e0136789. [CrossRef] [PubMed]

3. Hartman-Adams, H.; Banvard, C.; Juckett, G. Impetigo: Diagnosis and treatment. Am. Fam. Physician 2014, 90, 229-235. [PubMed]

4. Pereira, L.B. Impetigo-Review. Anais Brasileiros de Dermatologia 2014, 89, 293-299. [CrossRef] [PubMed]

5. Occelli, P.; Blanie, M.; Sanchez, R.; Vigier, D.; Dauwalder, O.; Darwiche, A.; Provenzano, B.; Dumartin, C.; Parneix, P.; Venier, A.G. Outbreak of Staphylococcal bullous impetigo in a maternity ward linked to an asymptomatic healthcare worker. J. Hosp. Infect. 2007, 67, 264-270. [CrossRef] [PubMed] 
6. Williamson, D.A.; Carter, G.P.; Howden, B.P. Current and emerging topical antibacterials and antiseptics: Agents, action, and resistance patterns. Clin. Microbiol. Rev. 2017, 30, 827-860. [CrossRef]

7. Rijnders, M.I.; Wolffs, P.F.; Hopstaken, R.M.; den Heyer, M.; Bruggeman, C.A.; Stobberingh, E.E. Spread of the epidemic European fusidic acid-resistant impetigo clone (eefic) in general practice patients in the south of the Netherlands. J. Antimicrob. Chemother. 2012, 67, 1176-1180. [CrossRef]

8. SanMiguel, A.J.; Meisel, J.S.; Horwinski, J.; Zheng, Q.; Grice, E.A. Topical antimicrobial treatments can elicit shifts to resident skin bacterial communities and reduce colonization by Staphylococcus aureus competitors. Antimicrob. Agents Chemother. 2017, 61, e00774-e00817. [CrossRef]

9. Gutiérrez, D.F.L.; Rodríguez, A.; García, P. Are phage lytic proteins the secret weapon to kill Staphylococcus aureus? mBio 2018, 9, e01923-e02017. [CrossRef]

10. Haddad Kashani, H.; Schmelcher, M.; Sabzalipoor, H.; Seyed Hosseini, E.; Moniri, R. Recombinant endolysins as potential therapeutics against antibiotic-resistant Staphylococcus aureus: Current status of research and novel delivery strategies. Clin. Microbiol. Rev. 2018, 31, e00071-e00117. [CrossRef]

11. Szweda, P.; Schielmann, M.; Kotlowski, R.; Gorczyca, G.; Zalewska, M.; Milewski, S. Peptidoglycan hydrolases-potential weapons against Staphylococcus aureus. Appl. Microbiol. Biotechnol. 2012, 96, 1157-1174. [CrossRef] [PubMed]

12. Love, M.J.; Bhandari, D.; Dobson, R.C.J.; Billington, C. Potential for bacteriophage endolysins to supplement or replace antibiotics in food production and clinical care. Antibiotics 2018, 7, 17. [CrossRef] [PubMed]

13. Fischetti, V.A. Bacteriophage endolysins: A novel anti-infective to control gram-positive pathogens. Int. J. Med. Microbiol. 2010, 300, 357-362. [CrossRef] [PubMed]

14. Cisek, A.A.; Dabrowska, I.; Gregorczyk, K.P.; Wyzewski, Z. Phage therapy in bacterial infections treatment: One hundred years after the discovery of bacteriophages. Curr. Microbiol. 2017, 74, 277-283. [CrossRef] [PubMed]

15. Zhang, L.; Li, D.; Li, X.; Hu, L.; Cheng, M.; Xia, F.; Gong, P.; Wang, B.; Ge, J.; Zhang, H.; et al. Lysgh15 kills Staphylococcus aureus without being affected by the humoral immune response or inducing inflammation. Sci. Rep. 2016, 6, 29344. [CrossRef] [PubMed]

16. Yang, H.; Zhang, H.; Wang, J.; Yu, J.; Wei, H. A novel chimeric lysin with robust antibacterial activity against planktonic and biofilm methicillin-resistant Staphylococcus aureus. Sci. Rep. 2017, 7, 40182. [CrossRef] [PubMed]

17. Totte, J.E.E.; van Doorn, M.B.; Pasmans, S. Successful treatment of chronic Staphylococcus aureus-related dermatoses with the topical endolysin staphefekt sa.100: A report of 3 cases. Case Rep. Dermatol. 2017, 9, 19-25. [CrossRef]

18. De Wit, J.; Totte, J.E.E.; van Mierlo, M.M.F.; van Veldhuizen, J.; van Doorn, M.B.A.; Schuren, F.H.J.; Willemsen, S.P.; Pardo, L.M.; Pasmans, S. Endolysin treatment against Staphylococcus aureus in adults with atopic dermatitis: A randomized controlled trial. J. Allergy Clin. Immunol. 2019, 27, 30691-30698. [CrossRef] [PubMed]

19. Oliveira, H.; Melo, L.; Santos, S.; Nóbrega, F.L.; Ferreira, E.; Cerca, N.; Azeredo, J.; Kluskens, L.D. Molecular aspects and comparative genomics of bacteriophage endolysins. J. Virol. 2013, 87, 4558-4570. [CrossRef]

20. Totté, J.; de Wit, J.; Pardo, L.; Schuren, F.; van Doorn, M.; Pasmans, S. Targeted anti-staphylococcal therapy with endolysins in atopic dermatitis and the effect on steroid use, disease severity and the microbiome: Study protocol for a randomized controlled trial (maas trial). Trials 2017, 18, 404. [CrossRef]

21. Kim, H.B.; Park, B.W. Phase iia Clinical Study of n-rephasin®sal200; ClinicalTrials.Gov Identifier: NCT03089697. Available online: https://clinicaltrials.gov/ct2/show/NCT03089697 (accessed on 10 July 2018).

22. Takemura-Uchiyama, I.; Uchiyama, J.; Kato, S.-I.; Ujihara, T.; Daibata, M.; Matsuzaki, S. Genomic and phylogenetic traits of Staphylococcus phages s25-3 and s25-4 (family myoviridae, genus twort-like viruses). Ann. Microbiol. 2013, 64, 1453-1456. [CrossRef]

23. Takemura-Uchiyama, I.; Uchiyama, J.; Kato, S.; Inoue, T.; Ujihara, T.; Ohara, N.; Daibata, M.; Matsuzaki, S. Evaluating efficacy of bacteriophage therapy against Staphylococcus aureus infections using a silkworm larval infection model. FEMS Microbiol. Lett. 2013, 347, 52-60. [CrossRef] [PubMed]

24. Gill, S.R.; Fouts, D.E.; Archer, G.L.; Mongodin, E.F.; Deboy, R.T.; Ravel, J.; Paulsen, I.T.; Kolonay, J.F.; Brinkac, L.; Beanan, M.; et al. Insights on evolution of virulence and resistance from the complete genome analysis of an early methicillin-resistant Staphylococcus aureus strain and a biofilm-producing methicillin-resistant Staphylococcus epidermidis strain. J. Bacteriol. 2005, 187, 2426-2438. [CrossRef] [PubMed] 
25. Kuroda, M.; Ohta, T.; Uchiyama, I.; Baba, T.; Yuzawa, H.; Kobayashi, I.; Cui, L.; Oguchi, A.; Aoki, K.; Nagai, Y.; et al. Whole genome sequencing of meticillin-resistant Staphylococcus aureus. Lancet 2001, 357, 1225-1240. [CrossRef]

26. Oyama, K.; Kawada-Matsuo, M.; Oogai, Y.; Hayashi, T.; Nakamura, N.; Komatsuzawa, H. Antibacterial effects of glycyrrhetinic acid and its derivatives on Staphylococcus aureus. PloS ONE 2016, 11, e0165831. [CrossRef] [PubMed]

27. Hisatsune, J.; Hirakawa, H.; Yamaguchi, T.; Fudaba, Y.; Oshima, K.; Hattori, M.; Kato, F.; Kayama, S.; Sugai, M. Emergence of Staphylococcus aureus carrying multiple drug resistance genes on a plasmid encoding exfoliative toxin b. Antimicrob Agents Chemother 2013, 57, 6131-6140. [CrossRef] [PubMed]

28. Imanishi, I.; Hattori, S.; Hisatsune, J.; Ide, K.; Sugai, M.; Nishifuji, K. Staphylococcus aureus penetrate the interkeratinocyte spaces created by skin-infiltrating neutrophils in a mouse model of impetigo. Vet. Dermatol. 2017, 28, e126-e127. [CrossRef]

29. Kawamura, Y.; Whiley, R.A.; Shu, S.E.; Ezaki, T.; Hardie, J.M. Genetic approaches to the identification of the mitis group within the genus Streptococcus. Microbiology 1999, 145, 2605-2713. [CrossRef]

30. Uchiyama, J.; Suzuki, M.; Nishifuji, K.; Kato, S.; Miyata, R.; Nasukawa, T.; Yamaguchi, K.; Takemura-Uchiyama, I.; Ujihara, T.; Shimakura, H.; et al. Analyses of short-term antagonistic evolution of Pseudomonas aeruginosa strain pao1 and phage kpp22 (myoviridae family, pb1-like virus genus). Appl. Environ. Microbiol. 2016, 82, 4482-4491. [CrossRef]

31. Wang, Q.; Garrity, G.M.; Tiedje, J.M.; Cole, J.R. Naive bayesian classifier for rapid assignment of rRNA sequences into the new bacterial taxonomy. Appl. Environ. Microbiol. 2007, 73, 5261-5267. [CrossRef]

32. Haritani, M.; Nakazawa, M.; Hashimoto, K.; Narita, M.; Tagawa, Y.; Nakagawa, M. Immunoperoxidase evaluation of the relationship between necrotic lesions and causative bacteria in lungs of calves with naturally acquired pneumonia. Am J Vet Res. 1990, 51, 1975-1981. [PubMed]

33. Castelino, M.; Eyre, S.; Moat, J.; Fox, G.; Martin, P.; Ho, P.; Upton, M.; Barton, A. Optimisation of methods for bacterial skin microbiome investigation: Primer selection and comparison of the 454 versus miseq platform. BMC Microbiology 2017, 17, 23. [CrossRef] [PubMed]

34. Bolyen, E.; Rideout, J.R.; Dillon, M.R.; Bokulich, N.A.; Abnet, C.; Al-Ghalith, G.A.; Alexander, H.; Alm, E.J.; Arumugam, M.; Asnicar, F.; et al. Qiime 2: Reproducible, interactive, scalable, and extensible microbiome data science. Peer] Preprints 2018, 6, e27295. [CrossRef] [PubMed]

35. Callahan, B.J.; McMurdie, P.J.; Rosen, M.J.; Han, A.W.; Johnson, A.J.; Holmes, S.P. Dada2: High-resolution sample inference from illumina amplicon data. Nat. Methods 2016, 13, 581-583. [CrossRef] [PubMed]

36. Flaherty, S.; Coffey, A.; Edwards, R.; Meaney, W.; Fitzgerald, G.F.; Ross, R.P. Genome of staphylococcal phage $\mathrm{k}$ : A new lineage of myoviridae infecting gram-positive bacteria with a low $\mathrm{g}+\mathrm{c}$ content. J. Bacteriol. 2004, 186, 2862-2871. [CrossRef] [PubMed]

37. Gu, J.; Xu, W.; Lei, L.; Huang, J.; Feng, X.; Sun, C.; Du, C.; Zuo, J.; Li, Y.; Du, T.; et al. Lysgh15, a novel bacteriophage lysin, protects a murine bacteremia model efficiently against lethal methicillin-resistant Staphylococcus aureus infection. J. Clin. Microbiol. 2011, 49, 111-117. [CrossRef] [PubMed]

38. Jun, S.Y.; Jung, G.M.; Yoon, S.J.; Oh, M.D.; Choi, Y.J.; Lee, W.J.; Kong, J.C.; Seol, J.G.; Kang, S.H. Antibacterial properties of a pre-formulated recombinant phage endolysin, sal-1. Int. J. Antimicrob. Agents 2013, 41, 156-161. [CrossRef] [PubMed]

39. Kawai, Y.; Mickiewicz, K.; Errington, J. Lysozyme counteracts $\beta$-lactam antibiotics by promoting the emergence of l-form bacteria. Cell 2018, 172, 1038-1049. [CrossRef]

40. Monteiro, R.; Pires, D.P.; Costa, A.R.; Azeredo, J. Phage therapy: Going temperate? Trends Microbiol. 2019, 27, 368-378. [CrossRef]

41. Cogen, A.L.; Nizet, V.; Gallo, R.L. Skin microbiota: A source of disease or defence? Br. J. Dermatol. 2008, 158, 442-455. [CrossRef]

42. Scharschmidt, T.C.; Fischbach, M.A. What lives on our skin: Ecology, genomics and therapeutic opportunities of the skin microbiome. Drug Discov. Today Dis. Mech. 2013, 10, e83-e89. [CrossRef] [PubMed]

43. Elias, P.M. The skin barrier as an innate immune element. Semin. Immunopathol. 2007, 29, 3-14. [CrossRef] [PubMed]

44. Alkilani, A.Z.; McCrudden, M.T.C.; Donnelly, R.F. Transdermal drug delivery: Innovative pharmaceutical developments based on disruption of the barrier properties of the stratum corneum. Pharmaceutics 2015, 7, 438-470. [CrossRef] [PubMed] 
45. Clokie, M.R.; Millard, A.D.; Letarov, A.V.; Heaphy, S. Phages in nature. Bacteriophage 2011, 1, 31-45. [CrossRef] [PubMed]

46. Filatova, L.Y.; Becker, S.C.; Donovan, D.M.; Gladilin, A.K.; Klyachko, N.L. Lysk, the enzyme lysing Staphylococcus aureus cells: Specific kinetic features and approaches towards stabilization. Biochimie 2010, 92, 507-513. [CrossRef] [PubMed]

47. Garg, T.; Rath, G.; Goyal, A.K. Comprehensive review on additives of topical dosage forms for drug delivery. Drug Deliv. 2015, 22, 969-987. [CrossRef] [PubMed]

48. Shen, Y.; Barros, M.; Vennemann, T.; Gallagher, D.T.; Yin, Y.; Linden, S.B.; Heselpoth, R.D.; Spencer, D.J.; Donovan, D.M.; Moult, J.; et al. A bacteriophage endolysin that eliminates intracellular streptococci. eLife 2016, 5, e13152. [CrossRef] [PubMed]

49. Fischetti, V.A. Lysin Therapy Offers New Hope For Fighting Drug-Resistant Bacteria. Available online: https:// www.rockefeller.edu/news/24920-lysin-therapy-offers-new-hope-fighting-drug-resistant-bacteria/ (accessed on 4 August 2019).

50. Jun, S.Y.; Jung, G.M.; Yoon, S.J.; Choi, Y.-J.; Koh, W.S.; Moon, K.S.; Kang, S.H. Preclinical safety evaluation of intravenously administered sal200 containing the recombinant phage endolysin sal-1 as a pharmaceutical ingredient. Antimicrob. Agents Chemother. 2014, 58, 2084-2088. [CrossRef] [PubMed]

51. Naik, S.; Larsen, S.B.; Gomez, N.C.; Alaverdyan, K.; Sendoel, A.; Yuan, S.; Polak, L.; Kulukian, A.; Chai, S.; Fuchs, E. Inflammatory memory sensitizes skin epithelial stem cells to tissue damage. Nature 2017, 550, 475-480. [CrossRef]

52. Pastagia, M.; Euler, C.; Chahales, P.; Fuentes-Duculan, J.; Krueger, J.G.; Fischetti, V.A. A novel chimeric lysin shows superiority to mupirocin for skin decolonization of methicillin-resistant and -sensitive Staphylococcus aureus strains. Antimicrob. Agents Chemother. 2011, 55, 738-744. [CrossRef]

53. Shimakura, H.; Uchiyama, J.; Saito, T.; Miyaji, K.; Fujimura, M.; Masuda, K.; Okamoto, N.; DeBoer, D.J.; Sakaguchi, M. Ige reactivity to hen egg white allergens in dogs with cutaneous adverse food reactions. Vet. Immunol. Immunopathol. 2016, 177, 52-57. [CrossRef] [PubMed]

54. Caubet, J.-C.; Wang, J. Current understanding of egg allergy. Pediatr. Clin. North Am. 2011, 58, 427-443. [CrossRef] [PubMed]

55. Piechowicz, L.; Garbacz, K.; Budzynska, A.; Dabrowska-Szponar, M. Outbreak of bullous impetigo caused by Staphylococcus aureus strains of phage type 3c/71 in a maternity ward linked to nasal carriage of a healthcare worker. Eur. J. Dermatol. 2012, 22, 252-255. [CrossRef] [PubMed]

56. Nguyen, T.A.; Wang, A.S.; Eichenfield, L.F.; Barrio, V. “Eb, or not eb?" Neonatal desquamative impetigo in a degloving pattern. Pediatr. Dermatol. 2016, 33, e147-e148. [CrossRef] [PubMed] 